\title{
Two Cases of Penetrating Injury of the Neck
}

\author{
Col PD Gupta*, Lt Col RN Verma ${ }^{+}$, Maj V Anand ${ }^{\#}$
}

MJAFI 2009; 65 : 371-372

Key Words : Penetrating injury; Neck

\section{Introduction}

$\mathrm{P}_{\mathrm{o}}^{\mathrm{e}}$ enetrating neck trauma has been a significant cause of morbidity and death. More than 4,000 cases of neck wounds were reported during American civil war with mortality rate of $15 \%$. The risk of death was reduced to $11 \%$ in World War I and 7\% in World War II [1]. We present two cases of penetrating neck injury (Grenade splinter-1, Stab-1) reporting to a mid zonal hospital in counter insurgency situation.

\section{Case 1}

A young serving soldier was admitted to the hospital on with history of grenade blast injury of right hand with multiple splinter injuries (right side of neck, right side of chest and right side of abdomen).

On examination, the patient was not dyspneic, had normal voice and was haemodynamically stable. There was one $(1 \times 1 \mathrm{~cm})$ splinter wound on right side of neck with minimal oozing, mild swelling and significant subcutaneous emphysema. A 1x1 cm splinter wound was also found on right side of chest without active bleeding. Clinical examination did not show any obvious haemo/ pneumothorax or intraabdominal free fluid.

On investigation the radiograph of the neck showed a radio-opaque shadow (splinter) lying on left side of midline, having crossed the midline (Fig.1). Chest radiograph confirmed absence of any air or fluid in right thoracic cavity. At this stage, in view of the splinter having crossed the midline and presence of subcutaneous emphysema without pneumothorax, a diagnosis of injury to aero-digestive tract was made. Since the patient did not have dyspnoea or alteration of voice, it was supposed that possibly the splinter has injured pharynx or oesophagus.

The patient was operated in view of penetrating injury of neck and blast injury right hand. When a nasogastric tube was passed to empty the stomach before induction of anesthesia (to prevent aspiration), air leak from the neck wound was noticed, confirming airway injury.

Exploration of the neck wound was done through a horizontal incision in the neck which revealed injury to both superior poles of thyroid gland with through and through penetration of larynx just below the vocal cords associated with injury to the laryngeal cartilages. A $6 \mathrm{~mm}$ splinter was lying near the left superior pole of thyroid. The operative procedure involved removal of splinter, ligation of both superior pedicles of thyroid (along with ligation of superior poles below the injury), repair of both lateral walls of larynx, tracheostomy with closure of wound over suction drain and debridement of right hand wound

The patient had smooth post-operative recovery. Tracheostomy was removed after 10 days and patient had no breathing difficulty or alteration of voice. The patient is under follow up and there is no breathing difficulty or change in voice eight months after injury.

The case highlights the fact that an innocuous looking splinter injury had damaged thyroid gland and lateral walls of the larynx. Patient had no dyspnoea or alteration of voice despite through and through penetrating injury of larynx

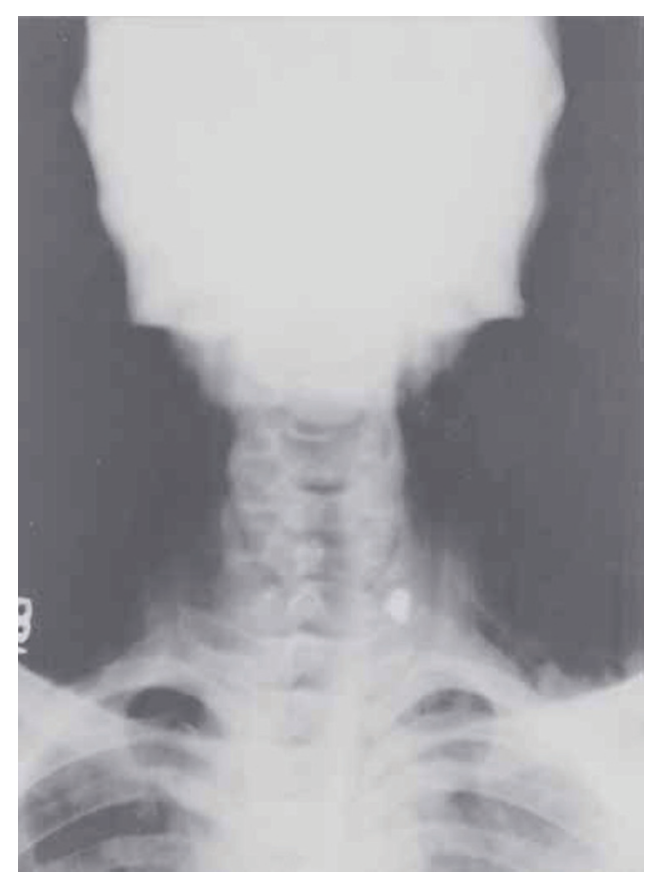

Fig. 1 : Neck radiograph showing splinter injury larynx

"Classified Specialist (Surgery), Armed Forces Clinic, Dalhousie Road, New Delhi-11. ${ }^{+}$Classified Specialist (Anaesthesia), MH, Dehradun (UP). " Graded Specialist (ENT), MH, Roorkee (UP).

Received : 26.06.07; Accepted : 15.05.09_E-mail:pdgupta18@gmail.com 


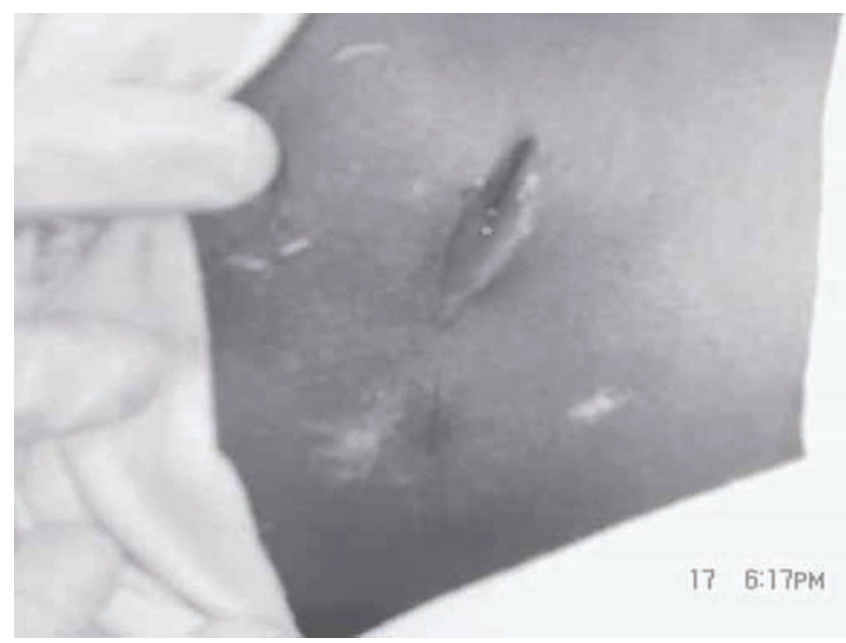

Fig. 2 : Stab injury neck

(both lateral walls). The patient had minimal oozing from the neck wound despite injury to thyroid gland which is a highly vascular structure.

\section{Case 2}

A 28 year old serving soldier was brought to this hospital with alleged history of self-inflicted stab wound on right side of neck with a small folding knife. Patient was found to have active bleeding from the wound and features of hypovolemic shock. Local examination showed $2 \mathrm{~cm}$ long deep, incised wound right side of neck, along with a $3 \mathrm{~cm}$ superficial wound (hesitation mark) (Fig. 2) and a small incised wound on right thumb.

The patient was immediately explored under general anaesthesia. During endotracheal intubation, blood was found inside the oropharynx along with a small incised wound in the lateral wall. There was severe edema of vocal cords and pharynx. Exploration revealed injury to right superior thyroid artery and wound tract reaching all the way to the pharyngeal wall. Exploration of tract, haemostasis (including ligation of bleeding superior thyroid artery), repair of pharyngeal wall and tracheostomy was carried out and patient had smooth post-operative recovery. Tracheostomy tube was removed on $14^{\text {th }}$ post-operative day and patient had normal voice.

The salient feature in this case was deep incised wound reaching pharyngeal wall caused by a small folding knife

\section{Discussion}

Laryngeal injuries are quite rare due to the protection offered to the laryngeal apparatus by mandible and cervical spine [2]. Laryngeal trauma estimates of $1: 15000$ to $1: 42000$ in patients attending accident and emergency services have been suggested [3]. There was a high incidence of injuries to the head and neck (up to $40 \%$ ) though they affected only $12 \%$ of the body surface area [4]. Stab wounds usually result in lesser degree of injury than missile injury [1].
Dysphonia (or aphonia), dyspnoea and stridor are common presenting features of laryngeal injury, others being hemoptysis, dysphagia, odynophagia, subcutaneous emphysema, loss of laryngeal prominence with cervical pain and tenderness [2]. However patients of laryngeal injury may appear to be normal. This deceptive appearance increases the risk of missed diagnosis and mismanagement which may result in serious airway and voice problems $[5,6]$.

Clinical signs are absent in 30\% neck explorations with positive findings and hence mandatory explorations of neck wounds is preferable. In laryngo-tracheal injuries, better results are obtained with early repair than delayed repair after three to five days [1]. Fogelman and Stewart revealed a mortality rate of $6 \%$ in patients who underwent immediate exploration versus 35\% mortality in patients who were treated conservatively or exploration was delayed [7].

Endotracheal intubation was difficult but successful in both these cases, though the most reliable method of securing airway in these circumstances is tracheostomy $[5,8,9]$. Finally, penetrating trauma of larynx has a better prognosis than blunt trauma [10].

\section{Conflicts of Interest}

None identified

\section{References}

1. Mary C Snyder. Penetrating injuries of neck Medicine. eMedicine / WEB MD / Otolaryngeal and facial plastic surgery; April 2006.

2. Kohli A, Bhadoria P, Bhalotra A, Anand R, Goyal P. An unusual laryngeal injury. Indian Journal of Anesthesia 2007; 51: 57-9.

3. Banerjee AS, Shaaban M, Gibson AL, Dilkes D. Post Traumatic Laryngeal Competence. Indian Journal of Otolaryngology and Head and Neck Surgery 2005; 57: 260-1.

4. Rustemeyer J, Kranz V, Bremerich A. Injuries in combat from $1982-2005$ with particular reference to those to the head and neck: a review. British Journal of Maxillofacial Surgery 2007; 45: 556-60.

5. Ikram M. Saleem N. Acute management of external laryngeal trauma. Ear, Nose and Throat Journal 2000; 79: 802-4.

6. Myers EM, Iko BO. The management of acute laryngeal trauma. J Trauma 1987: 27: 448-52.

7. Fogelman MJ, Stewart RD. Penetrating wounds of the neck. Am J Surg 1956; 91: 581-96.

8. Fuhrman GM, Stieg FH, Buerk CA. Blunt laryngeal trauma: Classification and management protocol. J Trauma 1990; 30: 87-92.

9. Schaefer SD, Close LG. Acute management of laryngeal trauma: Update. Ann Otol Rhinol Laryngol 1989; 98: 98-104.

10. Ahmed Subhy Al Sheikhly. Thyroid cartilage fracture: A case report and management review. Middle East Journal of Emergency Medicine 2005; 5. 25-7. 